\title{
Superconducting RF Linac Technology for ERL Light Sources
}

\author{
Christopher D. Tennant \\ Jefferson Laboratory, 12000 Jefferson Ave, Newport News, VA 23606
}

\begin{abstract}
Energy Recovering Linacs (ERLs) offer an attractive alternative as drivers for light sources as they combine the desirable characteristics of both storage rings (high efficiency) and linear accelerators (superior beam quality). Using superconducting RF technology allows ERLs to operate more efficiently because of the inherent characteristics of SRF linacs, namely that they are high gradient-low impedance structures and their ability to operate in the long pulse or CW regime. We present an overview of the physics challenges encountered in the design and operation of ERL based light sources with particular emphasis on those issues related to SRF technology. These challenges include maximizing a cavity's $Q_{0}$ to increase cryogenic efficiency, maintaining control of the cavity field in the presence of the highest feasible loaded $Q$ and providing adequate damping of the higher-order modes (HOMs). If not sufficiently damped, dipole HOMs can drive the multipass beam breakup (BBU) instability which ERLs are particularly susceptible to. Another challenge involves efficiently extracting the potentially large amounts of HOM power that are generated when a bunch traverses the SRF cavities and which may extend over a high range of frequencies. We present experimental data from the Jefferson Lab FEL Upgrade, a $10 \mathrm{~mA}$ ERL light source presently in operation, aimed at addressing some of these issues. We conclude with an outlook towards the future of ERL based light sources.
\end{abstract}

\section{INTRODUCTION}

Energy recovering linacs (ERLs) utilizing superconducting RF (SRF) linac technology hold tremendous potential as drivers for the next generation of light sources. Such drivers hold the promise of generating far superior beam quality (and hence radiation quality) than currently attainable in third generation light sources while operating in an economical and efficient manner. This paper will introduce the concept of energy recovery and the features that make it attractive as a driver for light sources. This leads naturally to a discussion of utilizing SRF technology in ERLs to take advantage of the continuous-mode and high gradient operation that can be achieved. However there exist several challenges in accelerating/decelerating high average current, short bunch length beams in an SRF environment. The primary purpose of this paper is to investigate these challenges in some detail. They include ensuring good cryogenic efficiency, operating a cavity with high loaded $Q$ in the presence of microphonic and Lorentz force detuning and recirculation path length errors. Another key issue, and to which a majority of the paper is devoted, is the importance of strong HOM damping and the consequences of not doing so - namely, the emergence of the multipass beam breakup (BBU) instability.

Currently, the most advanced ERL is the Jefferson Laboratory (JLab) $10 \mathrm{~kW}$ Free-electron laser (FEL) Upgrade shown schematically in Fig. 2 [Benson]. The FEL has achieved $10 \mathrm{~kW}$ of CW output laser power at a wavelength of 6 $\mu \mathrm{m}$ while the driver has accelerated and energy recovered up to $8 \mathrm{~mA}$ of average current to $145 \mathrm{MeV}$ ( $>1 \mathrm{MW}$ beam power) and up to $9 \mathrm{~mA}$ to $88 \mathrm{MeV}$. Because it is such a convenient testbed to explore many of the issues of SRF linac technology in ERLs, this paper will place a particular emphasis on work that has been performed at the FEL. 


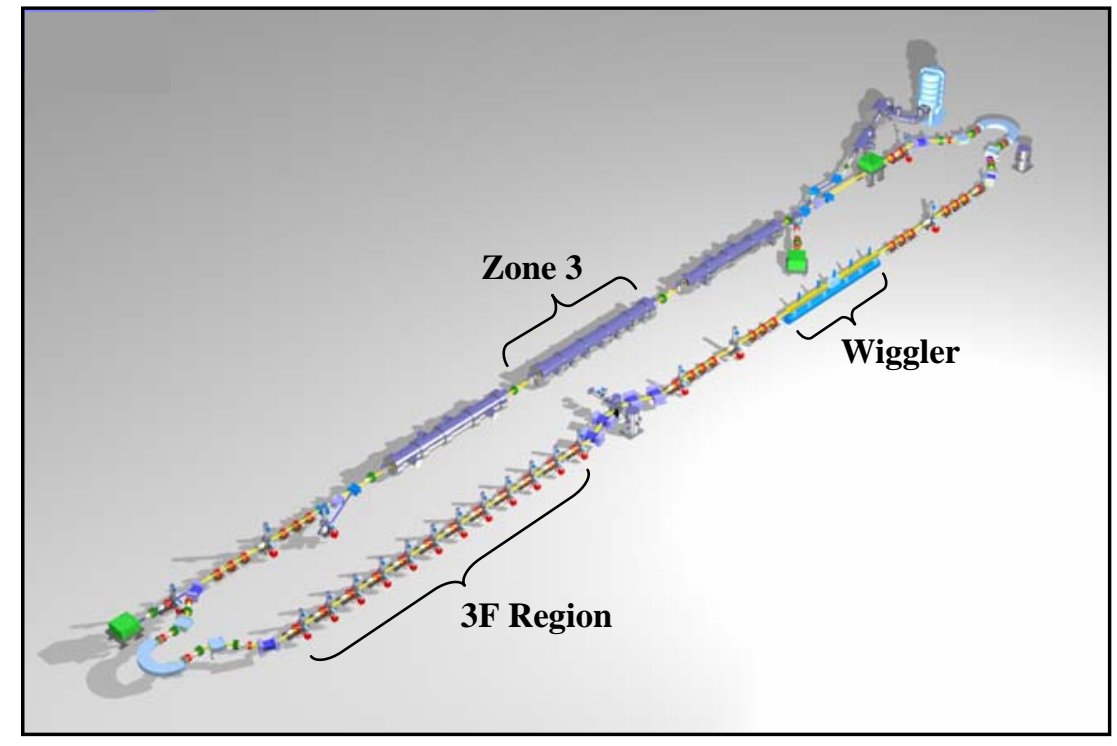

Figure 1: Layout of the Jefferson Laboratory FEL $10 \mathrm{~kW}$ Upgrade (the UV beam line which is currently under construction has been omitted).

\section{ENERGY RECOVERY LINACS}

The idea of energy recovery was first proposed in 1965 for use in a collider [Tigner]. While such a collider was never realized, energy recovery has found a niche as drivers for light sources. A schematic for a generic ERL based light source is given in Fig. 2. Electrons are generated in a high brightness injector, accelerated through a linear accelerator (linac) and then transported to a region where the desired radiation is generated (e.g. an undulator or an FEL). After performing their intended purpose, the electrons are returned to the linac $180^{\circ}$ out of phase with respect to the RF accelerating field for energy recovery. At the exit of the linac, the decelerated beam is now at approximately the injection energy and sent to a beam dump. In ERLs the decelerated beam cancels the beam loading effects of the accelerated beam. Therefore ERLs can, in principle, accelerate very high currents with only modest amounts of RF power. While energy recovery can be achieved in normal conducting or superconducting linacs, in this paper we assume the use of an SRF linac for reasons discussed in the following section.

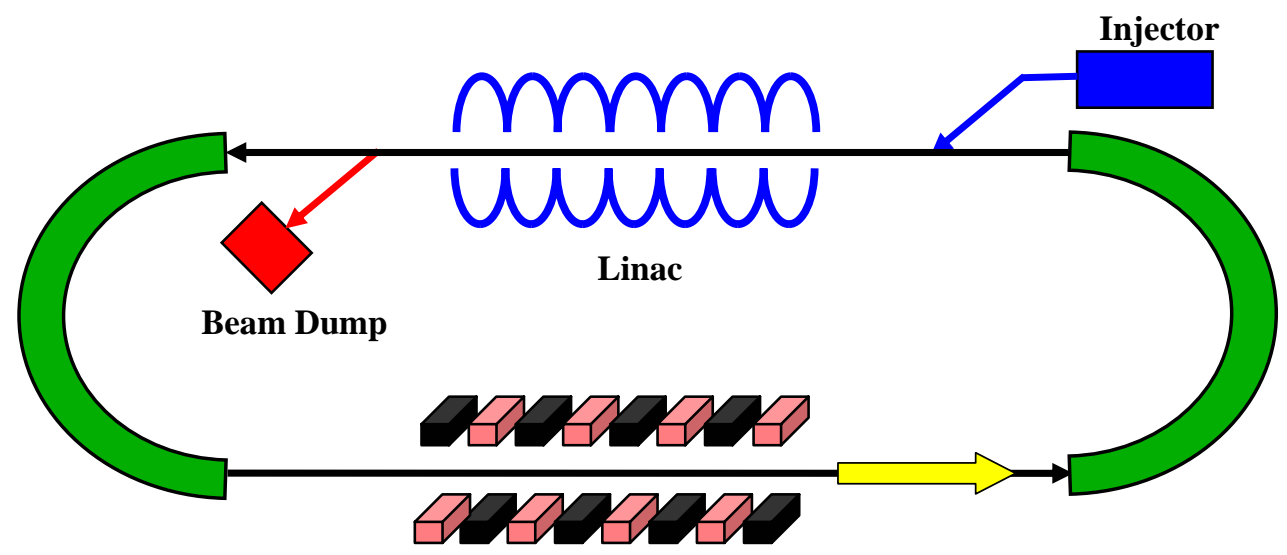

Photon Generator

Figure 2: Schematic of a generic energy recovering based light source [Lia EPAC04]. 
To appreciate the unique advantages of an ERL it is useful to consider the two traditional types of accelerators: linear accelerators and storage rings. Electron storage rings have been used for decades to meet the demands for beams with high average current while operating at high efficiency. However, one of the limitations of storage rings is the fact that the 6-dimensional phase space of the beam is determined by an equilibrium which sets an upper limit of the achievable beam quality. This equilibrium is determined by two competing processes; quantum excitation and radiation damping. On the other hand, linear accelerators can produce beam of exceptional quality, having small emittance and energy spread and the ability to generate very short bunches. However, one of the limitations of linacs is the prohibitive cost of RF power which in turn limits the amount of average current that can be accelerated. In an ERL, as previously mentioned, because the net current seen in the linac is negligible, high average currents can be accelerated economically. Furthermore, since the electron beam only exists in the accelerator for a short time (typically two passes), the equilibrium that is unavoidable in a storage ring does not have time to develop. Thus the beam quality in an ERL is determined, to a large extent, by the injector. This combination of high current capability and high beam quality make ERLs attractive as, among other things, drivers for oscillator FELs and synchrotron light sources [Lia EPAC].

ERL based FELs are capable of achieving unprecedented average laser power, high overall system efficiency (since the RF power consumption in the linac is nearly independent of beam current), and minimizing beam dump activation (since the final energy is relatively low). Synchrotron light ERLs (SL ERLs), are attractive because of their potential for producing radiation with superior quality compared to what is currently achievable in third generation synchrotron light sources. These parameters are possible due to the properties and flexibility of ERLs, such as, high average current (high average flux), small emittance (high average brightness) and a geometry that allows the insertion of long undulators. Furthermore, diffraction-limited round beams (full spatial coherence), small energy spread (high temporal coherence), and short electron bunches (sub-picosecond x-rays) allow for other highly desirable characteristics that can, in principle, be achieved in an ERL driver [White].

\section{SUPERCONDUCTING RF TECHNOLOGY}

While in principle there is nothing that prohibits the use of normal conducting RF cavities for energy recovery [BINP], we will find that SRF has clear advantages which have made it the technology of choice for nearly all ERL designs. The primary features that make it so attractive are the high quality factor, $Q_{o}$, and high accelerating gradient $V_{a c c}$, which can be achieved. The high $Q_{o}$ implies that the power dissipated is small which in turn allow for cavities to operate in a continuous-wave (CW) mode while maintaining high gradients. This is in stark contrast to normal conducting cavities where high fields can exist for only microseconds because of the substantial RF power required and because of issues related to providing adequate cooling to the cavity. In fact, operating copper cavities in CW mode is limited to gradients of less than $2 \mathrm{MV} / \mathrm{m}$. As an added benefit, the support of high gradients relaxes the requirements for the length of the linac.

Another important advantage of SRF cavities is the ability to increase the beam aperture. While this has the adverse effect of decreasing the $(R / Q)$ of the fundamental mode, in some sense this effect can be absorbed by the extremely high $Q_{o}$, since the quantity of interest is $(R / Q) Q_{o}$. A larger aperture ensures increased beam quality by reducing the shortrange wakefields and thereby reducing emittance growth along the linac, it ensures greater beam stability by reducing the impedance of HOMs, and reduces beam loss. Better RF control and stability can also be achieved in SRF cavities. All of these benefits combine to make it possible to accelerate high average current.

Furthermore, in SRF cavities there is a high RF power to beam power efficiency. To see this, we introduce the concept of a multiplication factor as a way to quantify the efficiency of an ERL, which is expressed as [Un]

$$
\kappa=\frac{P_{b}}{P_{R F}} \approx \frac{I_{b} E_{\text {final }}}{I_{b} E_{\text {inj }}+P_{R F, \text { linac }}}
$$

where $P_{b}$ is the power of the beam and $P_{R F}$ is the power required to operate the RF cavities. For a machine operating in the regime of perfect energy recovery (the accelerated and energy recovered beams cancel), $P_{R F}$ consists of two terms: the first is the power required to accelerate a beam current $I_{b}$ in the injector (which is not energy recovered) to an energy $E_{i n j}$ and $P_{R F \text {,linac }}$ is the power required to establish the accelerating fields in the cavity. Note that this last term is independent of beam current. The key is in this term which is inversely proportional to $Q_{L}$ and which, in SRF cavities, is typically three orders of magnitude greater than in normal conducting cavities. Consequently, ERLs based on SRF 
technology have a $\kappa$ value of several hundred times greater than ERLs based on normal conducting technology [Ann Rev]. Therefore, ERLs based on SRF technology yield a machine that operates both economically and efficiently.

\section{CHALLENGES FOR SUPERCONDUCTING RF}

Accelerating cavities in storage rings can accommodate high average current beams but the bunch is sufficiently long that only lower frequency HOMs are excited and of concern. On the other hand, recirculating linacs, such as JLab's Continuous Electron Beam Accelerator Facility (CEBAF) [CEBAF], accelerate relatively low currents $(100 \mu \mathrm{A})$ with very short bunches. Future ERL drivers for light sources are unique in that they require accelerating high average current (on the order of $100 \mathrm{~mA}$ or more) and very short bunches, thereby presenting unique challenges.

There exists in any ERL, two extreme SRF regimes. The injector, which is not energy recovered, is dominated by very strong beam loading whereas the linac sees virtually no beam loading effects. This paper will address the particular challenges for the latter regime.

\subsection{Power Dissipation}

Energy recovering linacs based on SRF technology are being considered for a wide variety of applications; from electron-ion colliders [ELIC][eRHIC], to light sources to electron coolers [eCOOL]. There exists, even amongst the ERL based light sources, a variety of applications. The two extremes are FELs, which typically desire moderate beam energies (100 - $200 \mathrm{MeV}$ ) and very high average current (1 A), and SL ERLs which also desire a substantial average current $(\geq 100 \mathrm{~mA}$ ) but want to push the energy to several GeV. Depending on the intended use, SRF parameters from one machine to another can differ substantially. Designing an SRF linac requires a process of optimization with regards to the relevant figures of merit and often compromises must be made.

While many factors come into play, which depend on the detailed nature of the accelerator in question, in terms of cost optimization for operating a CW SRF linac, the optimal gradient lies somewhere in the (12 - 20) MV/m range [Schneider PAC03] [Liepe]. Given that, the next step is to determine the optimal fundamental frequency at which to operate. According to the Bardeen, Cooper, and Schreiffer (BCS) theory, the surface resistance is proportional to the frequency squared [Padam]. At high frequencies, where the surface resistance becomes large, increased losses occur. On the other hand, at lower frequencies the increased cavity size increases power consumption [Liepe]. Therefore optimization with regards to other parameters must be considered. For instance, for Cornell University's proposed SL ERL, cavities will be operated at $1300 \mathrm{MHz}$ whereas future upgrades of JLab's FEL will utilize cavities operating at $750 \mathrm{MHz}$. Unfortunately there are no universal, ideal figures of merit for SRF cavity design. But rather, each machine and its intended application must be considered individually.

Continuous-wave operation of SRF cavities requires that cryogenic losses be minimized. The power dissipated on the cavity wall is given by

$$
P_{\text {diss }}=\frac{V_{a c c}^{2}}{(R / Q) G} R_{s}
$$

where $V_{\text {acc }}$ is the accelerating gradient, $(R / Q)$ is the shunt impedance, $R_{S}$ is the surface resistance and $G$ is the geometry constant (note that $Q_{o}$ is defined as $R_{s} / G$ ). Clearly, the goal is to maximize $(R / Q)$ and $G$ for the accelerating mode. Decreasing the iris diameter leads to an increase in the quantity $(R / Q) G$, however this must be weighed against the benefits of lowering the loss factor by increasing the iris diameter [Jacek03]. That is to say, a small iris diameter minimizes cryogenic losses and hence operating costs, but if it comes at the expense of beam stability, then compromises are required.

To get an idea of the other parameters that must be optimized, consider the following. The required wall plug (AC) power is proportional the accelerating gradient squared and inversely proportional to $Q_{o}$. Thus for a given accelerating gradient, we want to maximize $Q_{o}$. However, $Q_{o}$ depends on the surface resistance, which in turn, according to the BCS theory is strongly dependent on the operating bath temperature. $R_{B C S}$ decreases dramatically as the temperature is lowered, but on the other hand, the choice of operating temperature is dependent on the Carnot efficiency which is given by 


$$
\eta_{c}=\frac{T}{300-T}
$$

and decreases with lower temperatures.

Perhaps the relationships between the various parameters are best illustrated with some examples. Consider a cavity with a gradient of $15 \mathrm{MV} / \mathrm{m},(R / Q)$ of $1000 \Omega / \mathrm{m}$ and $Q_{o}$ of $1 \times 10^{10}$. This corresponds to losses of approximately 20 $\mathrm{W} / \mathrm{m}$. The net refrigerator efficiency is given by the product of the Carnot efficiency and technical efficiency, $\eta_{t}$, which is typically around $20 \%$ for large refrigerator systems [Padam]. With a net refrigerator efficiency of 0.0014 at $2.2 \mathrm{~K}$, the required wall plug power to operate the cavity is $16 \mathrm{~kW} / \mathrm{m}$. Now consider the same cavity, but where we have been able to achieve a $Q_{o}$ of $6 \times 10^{10}$ by lowering the operating bath temperature to $1.8 \mathrm{~K}$. The resulting refrigerator efficiency becomes 0.0012 and the required wall plug power is $3 \mathrm{~kW} / \mathrm{m}$. Or put another way, given the same wall plug power as before $(16 \mathrm{~kW} / \mathrm{m})$, using these new parameters, we could, in principle, operate a cavity at $~ 35 \mathrm{MV} / \mathrm{m}$.

\subsection{High Loaded $Q$ Operation}

Because of the high Qs achieved in SRF cavities, bandwidths are on the order of several $\mathrm{Hz}$, which makes the resonance frequency extremely sensitive to perturbations. We consider two processes in particular that act to shift, or detune, the frequency. They are microphonic vibrations and Lorentz force detuning.

\subsubsection{Microphonics}

Microphonics describes the modulation of a cavity's resonance frequency by external mechanical disturbances. Because of the small bandwidths (high Qs) of resonant modes SRF cavities are particularly susceptible to microphonic vibrations. These perturbations cause the resonance frequency to shift, which in turn causes the phase and amplitude of the cavity field to change [Curt TN]. Left uncontrolled, these changes will severely degrade beam quality (i.e. energy spread) and lead to beam loss. Sources of these vibrations from the accelerator environment include vacuum pumps, where the vibrations are transferred by the beam pipe, from external vibration sources (vehicular traffic and machinery) where they are transferred through the ground and supports, and from the pumps and compressors of the cryogenic plan where the vibrations are transferred along helium transfer lines [Schilcher][Davis]. Microphonics can also originate from the mechanical resonances of the cavity itself which can be excited by any of the aforementioned sources [Davis].

Microphonics plays an especially important role in ERLs. This can most clearly be understood by considering the power required to maintain a constant field in an overcoupled cavity $\left(\beta=Q_{d} / Q_{e x t}>1\right)$ with no beam loading (perfect energy recovery) and in the presence of microphonics [Qext Opt]:

$$
P_{g}=\frac{V_{a c c}^{2}}{4(R / Q) Q_{e x t}}\left\{1+\left[\frac{2 \delta f}{f_{o}} Q_{e x t}\right]^{2}\right\}
$$

where $V_{a c c}$ is the cavity gradient, $(R / Q)$ is the shunt impedance, $\delta f$ is the peak-to-peak microphonics detuning and $f_{o}$ is the fundamental frequency. Given these cavity parameters, the goal is to optimize the external $Q$ such that the power is minimized. The optimal $Q_{e x t}$ is given by

$$
Q_{\text {ext,opt }}=\frac{f_{o}}{2 \delta f}
$$

and it follows that the minimum power required to operate the cavity is

$$
P_{g, o p t}=\frac{V_{a c c}^{2}}{(R / Q)} \frac{\delta f}{f_{o}} .
$$


Thus, for ERLs, where the beam loading is negligible, the required RF power is determined by the power required for phase and amplitude control of cavities whose resonance frequency deviates from the system's nominal frequency.

Consider as an example the JLab FEL Driver, and in particular, the second of the three cryomodules (henceforth referred to as Zone 3). The cryomodule consists of eight 7-cell cavities and, prior to a vacuum incident in the spring of 2005, was able to provide $15 \mathrm{MV} / \mathrm{m}$ accelerating gradient. The shunt impedance and external $Q$ are $960 \Omega / \mathrm{m}$ and $2 \mathrm{x}$ $10^{7}$, respectively. Using these parameters, the required RF drive power as a function of $Q_{\text {ext }}$ for several peak detuning frequencies is shown in Fig. 3. From this plot, the motivation for minimizing microphonic levels and pushing the external $Q$ is clear - it leads to a significant reduction in the RF drive power. The average peak-to-peak microphonics in the Zone 3 module was measured to be is $7.5 \mathrm{~Hz}$ [Davis]. Achieving these low microphonic levels was due in large part by making the string assembly stiffer. This has the effect of pushing the cavity's intrinsic mechanical frequencies higher where they are less susceptible to resonant excitation by the microphonic sources listed above.

If one can optimize the $Q_{e x t}$ to the extent that $P_{g}$ is less than $1 \mathrm{~kW}$ per cavity, then there are tremendous implications with regard to RF sources, where one can now consider using solid state amplifiers rather than inductive output tubes (IOTs), for example [Curt TN][Liepe05]. This is clearly illustrated in Fig. 4 which compares the RF driver power required during energy recovery at the JLab FEL for a loaded $Q$ of $2 \times 10^{7}$ and for $1.2 \times 10^{8}$. While this may not be a major issue in an ERL based FEL where energies are typically on the order of 100-200 MeV, for SL ERLs which are designed to operate at several GeVs, this would constitute a huge savings in cost.

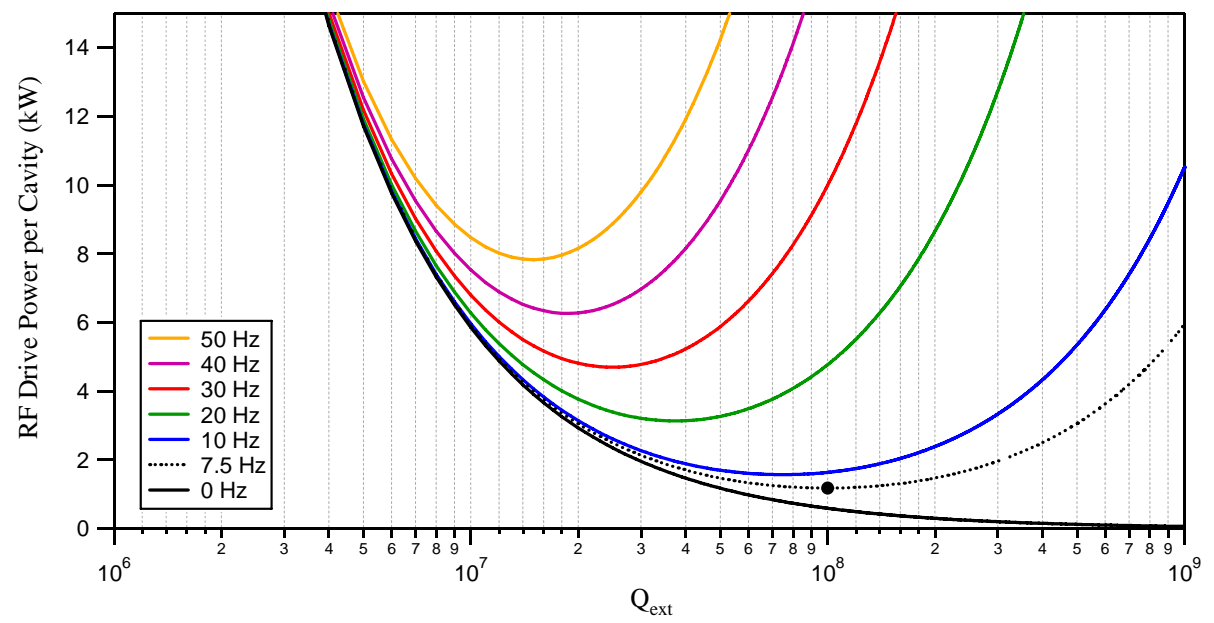

Figure 3: RF drive power as a function of $Q_{e x t}$ for several values of peak-to-peak microphonics detuning. The dashed line $(7.5 \mathrm{~Hz})$ indicates the average microphonics as measured in the eight 7-cell cavities in the FEL Zone 3 cryomodule. The black marker indicates denotes the external $Q\left(1 \times 10^{8}\right)$ at which the power is minimized $(1.2 \mathrm{~kW})$.
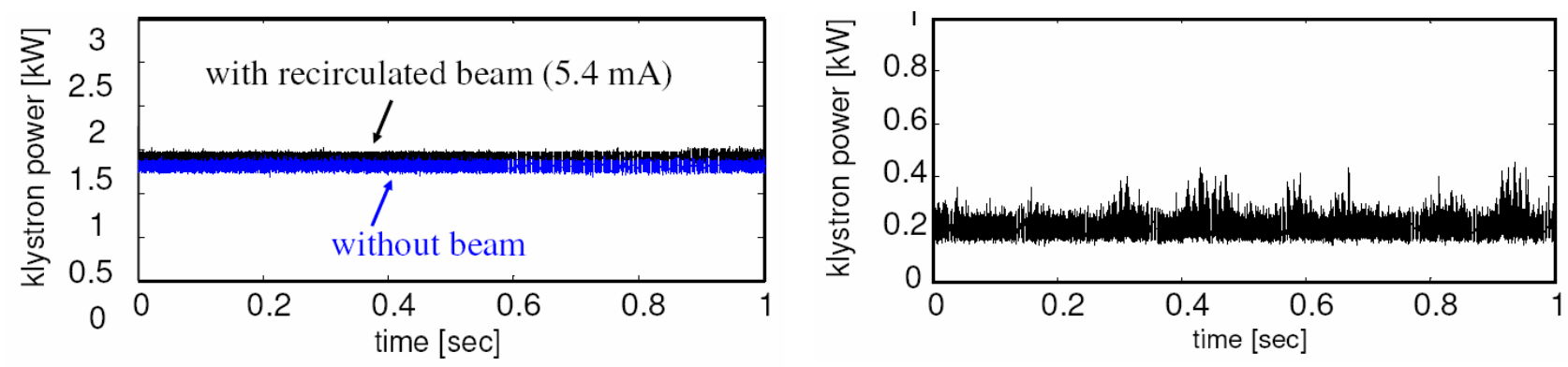

Figure 4: (Left) RF drive power required to operate a cavity with $Q_{L}=2 \times 10^{7}$ with and without energy recovery and (Right) RF drive power required to operate the same cavity with $5 \mathrm{~mA}$ with energy recovery and with the loaded increased to $1.2 \times 10^{8}$ [Liepe05]. 


\subsubsection{Lorentz Force Detuning}

Achieving a high accelerating gradient necessarily requires high electromagnetic fields in the SRF cavity. However, these high fields cause strong Lorentz forces which act to deform the cavity wall and lead to frequency shifts. The presence of very high Qs (narrow bandwidths) in conjunction with the relatively thin Niobium walls of the cavity, make it particularly susceptible to Lorentz force detuning. The static Lorentz force detuning is gradient-dependent and given by

$$
\Delta f_{L}=-K_{L} \cdot V_{a c c}^{2}
$$

where $K_{L}$ is the constant of proportionality and determined from measurements. For instance $K_{L}$ was measured to be 1 $\mathrm{Hz} /(\mathrm{MV} / \mathrm{m})^{2}$ in Zone 3 of the FEL [Davis]. While Lorentz force detuning is particularly important in pulsed linac structures, it is also of concern in ERLs, especially during field start-up (such as in a fault recovery). Because the cavity is making the transition from zero gradient to very high gradient, the cavity can detune over many bandwidths.

In 2004, a collaboration between JLab and Cornell University was established to test Cornell's new digital lowlevel RF (LLRF) system on a high $Q_{e x t}$ cavity in JLab's ERL FEL. One aspect of that work was to demonstrate rapid RF cavity recovery using a piezoelectric tuner. This was demonstrated at the FEL using a cavity with a $Q_{L}$ of $1.2 \times 10^{8}$ [Liepe05] [Curt TN]. The cavity was operated with a gradient of $12 \mathrm{MV} / \mathrm{m}$ where the corresponding Lorentz detuning is $144 \mathrm{~Hz}$, which corresponds to 12 bandwidths. The new LLRF system in conjunction with the piezoelectric tuner allowed the cavity to be ramped up to high gradient in less than 1 second. The plot of the gradient during this turn-on is shown in Fig. 5. This is to be contrasted to the minute or so, required to track and correct the detuning using the mechanical tuner which is slower and has considerably less resolution $(100 \mathrm{~Hz}$ versus the $1 \mathrm{~Hz}$ resolution of the piezoelectric [Deylayen99]). In addition to active compensation of Lorentz detuning with piezoelectric tuners [piezo1][piezo2], an example of passive compensation is to stiffen the cavities (e.g. with stiffener rings between cells) to reduce the deformations (and hence frequency shift) caused by the Lorentz force.

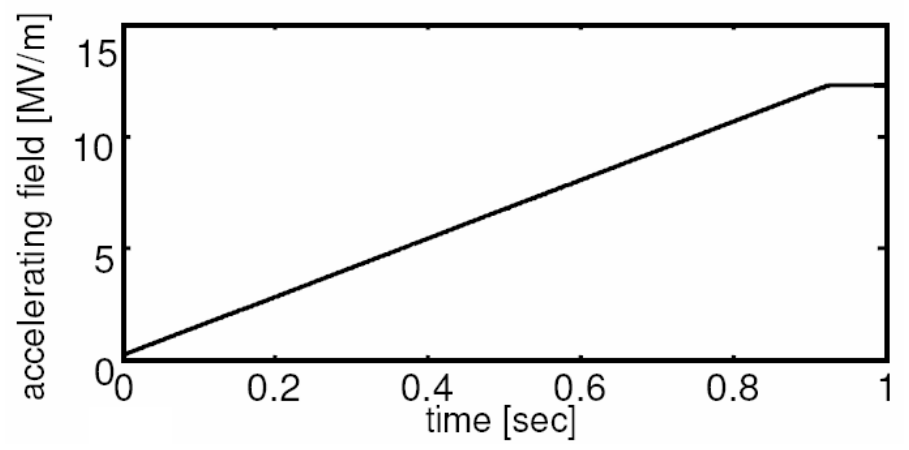

Figure 5: Demonstration of fast RF recovery using piezoelectric tuner in a cavity with $Q_{L}=1.2 \times 10^{8}$ [Liepe05].

\subsubsection{Random Beam Loading}

Thus far we have assumed that the energy recovery process is perfect in the sense that the accelerating and decelerating beams cancel and the beam loading effects in the cavity are negligible. However, errors in recirculation path length can lead to incomplete cancellation of the two beams and result in a net beam loading [Liepe PAC03]. For a GeV-class, $100 \mathrm{~mA}$ ERL, even a small path length error can translate into a substantial increase in the required RF drive power. Therefore, control of this so-called random beam loading is important to minimize the drive power.

\section{HOM DAMPING AND EXTRACTION}


While it is desirable to have cavities with high $Q_{o}$ and $Q_{L}$ for the fundamental mode, both of which can be achieved in SRF cavities, an unfortunate consequence is the presence HOMs with very high Qs. This requires strong HOM damping to avoid issues with beam instabilities. Recirculating linacs, and ERLs in particular, are more susceptible to these instabilities because they can support currents approaching or exceeding the threshold of the instabilities. The instability of greatest concern is transverse, multipass, multibunch beam breakup [Gluck].

\subsection{Multipass Beam Breakup}

A beam bunch will excite dipole HOMs in a cavity if it passes through the cavity off-axis. The magnetic field of the excited mode then acts to deflect the following bunches. The kick produced by the mode is translated into a transverse displacement at the cavity after recirculation. Thus, the recirculated beam constitutes a feedback which can cause the voltage of the HOMs to grow. This instability has been observed in the JLab FEL Driver and has provided a convenient testbed to investigate beam breakup [CT PRST].

For a two-pass accelerator with a single accelerating cavity containing a single dipole HOM with arbitrary polarization, $\alpha$, the threshold current is given by the expression

$$
I_{t h}=-\frac{2 c p_{b}}{e M^{*}(R / Q) Q k \sin \left(\omega T_{r}\right)}
$$

where

$$
M^{*} \equiv M_{12} \cos ^{2} \alpha+\left(M_{14}+M_{32}\right) \sin \alpha \cos \alpha+M_{34} \sin ^{2} \alpha
$$

and $p_{b}$ is the beam momenta at the cavity, $(R / Q) Q$ is the impedance of the mode, $k$ is the wavenumber and $T_{r}$ is the recirculation time [EP PRST]. Despite the simplicity of the model, it has proven to be very accurate in describing the effects of BBU in the FEL and for gaining physical insight into the instability. For example, in terms of the relevant HOM parameters, the threshold current scales as

$$
I_{t h} \propto \frac{1}{(R / Q) Q \omega}
$$

and the necessity for strong HOM damping is clear. Work at several institutions is being performed to address, among other issues, ways of providing strong HOM damping.

In general, one can add more loop couplers, waveguide couplers and/or open beam pipes to allow HOMs to propagate to the beam pipe and use broadband absorbers to provide damping of these modes. Increasing the beam pipe aperture in conjunction with lowering the RF frequency reduces the threat of short- and long-range wakefields which ensures greater beam stability [Padam]. Note that the shunt impedance of the fundamental will decrease and therefore increase the refrigerator load due to increased power dissipation, however with SRF, the decrease is not substantial compared to the gain in beam stability. It is worthwhile to briefly survey some of the fundamental parameters for three high-current SRF cavity designs being developed for use in different ERL applications.

At JLab a $1 \mathrm{~A}$ module is being developed for use in a driver for a high power (1 MW), compact FEL [Rimmer]. The module consists of six 5-cell cavities operating at $750 \mathrm{MHz}$. The cavity has a relatively large iris $(140 \mathrm{~mm}$ diameter) which will allow good clearance for beam halo. HOM damping is accomplished with waveguide couplers at both ends of the cavity and the HOM power is extracted at ambient temperature with water cooled ceramic HOM loads.

Cornell University is developing the technology required for their proposed $5 \mathrm{GeV}, 100 \mathrm{~mA}$ ERL based light source [Phase1]. The choice of technology is based on a 7-cell cavity operating at $1300 \mathrm{MHz}$. To achieve strong HOM damping, these cavities will be equipped with 6 HOM loop couplers per cavity. In order to minimize the possibility of trapped modes, the beam tube at one end will be enlarged to create an asymmetric cavity. Ferrite ring-absorbers (at 80 K) will be placed between cavities and damp the propagating TM monopole and dipole modes [Liepe][Barstow]. Brookhaven National Laboratory (BNL) is also actively involved in developing high current SRF cavities for electron cooling [cooling]. Like Cornell, they will increase the beam pipe diameter to allow HOMs to propagate to a ferrite ringabsorber [Calaga04]. 
A novel design worth mentioning is the concept of a superstructure [Jacek99]. A superstructure is a chain of several multi-cell cavities connected by $\lambda_{\mathrm{RF}} / 2$ long tubes. Since the beam loading in the cavity of an ERL is nearly zero, the required RF power per cell is quite small. Thus, in principle, many cavities can be serviced by a single fundamental power coupler (FPC). Having more cavities per FPC, and hence a less complex RF power distribution system, translates into substantial cost savings. However, because of the longer structure, care must be taken to extract and adequately damp trapped modes.

Since such strong HOM damping has yet to be demonstrated in an ERL, we will consider operational experiences with the JLab FEL. While in comparison to the proposed ERL light sources, the $10 \mathrm{~mA}$ of average current in the FEL seems modest, nevertheless, to date, this is the highest average current that has been successfully energy recovered in an SRF linac.

With the FEL configured for $88 \mathrm{MeV}$ energy operation and with nominal optics, the threshold current for the BBU instability occurred at approximately $2 \mathrm{~mA}$. By monitoring the HOM power from each cavity individually, we could determine that the mode causing the instability was contained in cavity 7 of Zone 3. Furthermore, by measuring the HOM voltage from this cavity during beam breakup, we could compute (via a FFT of the signal) that the frequency causing the instability was $2106 \mathrm{MHz}$. This agreed with simulation results which predicted that this HOM would produce the lowest threshold current. Incidentally, this mode has a measured loaded $Q$ of $6.1 \times 10^{6}$ and shunt impedance (from MAFIA simulations) of $5.7 \Omega / \mathrm{cm}^{2}$. The following discussions summarize two categories of beam suppression techniques that were exercised in the FEL. The first involves methods that require a modification of the electron beam optics and the second are methods that provide further damping of selected HOMs.

\subsection{Beam Optical Suppression}

Methods to manipulate the transverse beam optics in such a way as to suppress BBU were first discussed in 1980 [RandS]. We experimentally investigated the effectiveness of several of these methods in the FEL. Specifically, we demonstrated the ability to raise the threshold current by use of point-to-point focusing, a local reflector and a pseudoreflector. Each of these is discussed in more detail below.

\subsubsection{Point-to-Point Focusing}

With a judicious change in the phase advance, one can achieve point-to-point focusing $\left(M_{12}\right.$ or $\left.M_{34}=0\right)$ at the location of an unstable mode so that an HOM-induced kick on the first pass results in a zero displacement on the second pass [DD]. This precludes the beam from depositing more energy to the HOM. Such an adjustment of the recirculation optics is routinely used at the JLab FEL to increase the BBU threshold current. By all indications the $2106 \mathrm{MHz} \mathrm{HOM}$ is a vertically polarized mode. To change the vertical phase advance, the strength of four quadrupoles in the $3 \mathrm{~F}$ region were changed from their nominal setpoint in steps of $100 \mathrm{G}$ from $-200 \mathrm{G}$ to $+300 \mathrm{G}$ while measuring the threshold current at each point. The effect was to decrease the threshold current from below $1 \mathrm{~mA}(-200 \mathrm{G})$ to stabilizing the mode $(+300 \mathrm{G})$. The fact that the mode was eventually stabilized indicates that the $M_{34}$ element of the recirculation matrix from the unstable cavity back to itself changed sign. Fig. 6 shows the threshold current as a function of the change in quadrupole settings. The threshold exhibits a $1 /$ sin dependence which is a explained by the fact that the $M_{34}$ element of the recirculation matrix is proportional to $\sin (\Delta \psi)$ where $\Delta \psi$ is the change in phase advance for a recirculation. Note that while this technique has been shown to be very beneficial at the FEL, point-to-point focusing can not be arranged for each individual cavity in an extended accelerator with many cavities. Hence care should be taken when applying this method to large-scale ERLs with extended linacs. 


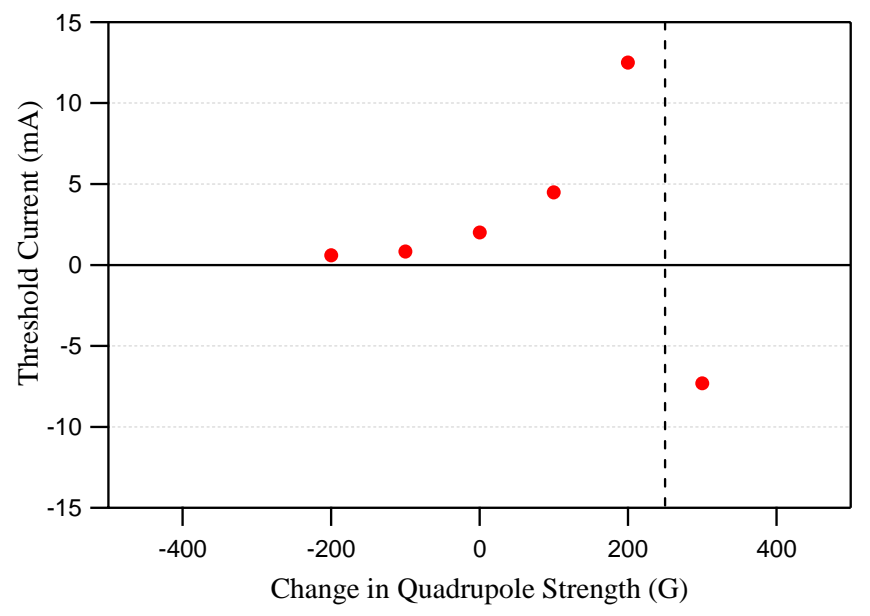

Figure 6: A plot of the threshold current due to the $2106 \mathrm{MHz} \mathrm{HOM}$ versus the change in four quadrupole strengths in the $3 F$ region of the JLab FEL. Depending on the quadrupole settings the threshold current could be decreased to less than $1 \mathrm{~mA}$ or completely stabilized (indicated by a negative threshold current).

\subsubsection{Local Reflector}

The idea behind implementing a local reflector is to map a BBU-induced horizontal kick into the vertical plane, and likewise to map a BBU-induced vertical kick to the horizontal plane. The action of the reflector produces a fullycoupled recirculation matrix from the unstable cavity back to itself which takes the form of

$$
\left(\begin{array}{ll}
0 & A \\
B & 0
\end{array}\right)
$$

where each element represents a 2x2 matrix. A practical implementation of a local reflector using skew-quadrupoles has been non-invasively embedded in the 3F region of the FEL Upgrade Driver [SQEEM]. When activated, the reflector interchanges the horizontal and vertical phase spaces, imaging each identically into the other. To see its effect on the threshold current, we first established that the threshold current without the reflector was $1.8 \mathrm{~mA}$ by direct observation. The reflector was then activated and a beam-transfer function (BTF) measurement [CDT05] performed to extract the threshold current with the result that the new threshold current was $9 \mathrm{~mA}$ - an increase by a factor of 5.

\subsubsection{Pseudo-Reflection}

If the $2 \times 2$ block matrix $B$ in $(10)$ is equal to $-B$, the transformation matrix

$$
\left(\begin{array}{cc}
0 & A \\
-B & 0
\end{array}\right)
$$

describes what we will call a pseudo-reflection because of its similarities to matrix (10). The motivation for generating a pseudo-reflection is that in some instances more effective damping can be achieved if the off-diagonal matrices are of opposite sign. The existing local reflector embedded in the FEL recirculator can, in principle, be made to produce such a transfer matrix by an appropriate modification of the optics [Rotate]. We attempted to produce such a pseudo-reflection, however, we do not know for certain the form of the transfer matrix created. We then followed the usual procedure of comparing the extrapolated threshold current from the BTF measurement with the threshold current with nominal, decoupled beam optics. The result of these measurements for the $2106 \mathrm{MHz} \mathrm{HOM}$ shows that the mode can be stabilized (i.e. the threshold current is well above $10 \mathrm{~mA}$ ) when the pseudo-reflector is activated. 


\subsection{Q-Damping}

Providing sufficient damping of the HOM quality factors is the primary role of the HOM couplers. However, in the event that the HOMs need further damping, two methods were developed and successfully exercised in the FEL. Initially, these were developed to provide a relatively fast and easy way to increase the threshold current.

\subsubsection{Narrow-band, Cavity-based Feedback}

The idea of the feedback is as follows; couple power from one of the cavity's HOM ports and using a narrowband filter select the HOM frequency of interest, shift the signal $180^{\circ}$ in phase, amplify the signal and then return it to the cavity through the same HOM port. The gain of loop is adjusted to be nearly unity by a combination of a variable attenuator and a low power amplifier. With careful tuning of the phase and gain of the feedback loop the $Q$ of the 2106 MHz HOM could be decreased by a factor of 20. However for practical purposes, damping the $Q$ by a factor of 10 was the best that could be achieved as the circuit becomes sensitive to external disturbances beyond this point. Since the threshold current is inversely proportional to the $Q$ of the mode (see Eqn. (8)), we expect that a factor of 5 decrease in the $Q$ will result in a corresponding increase in the threshold current. This is, approximately, what we observe experimentally. The threshold current was determined to be approximately $2 \mathrm{~mA}$ without the damping circuit and nearly $8 \mathrm{~mA}$ with the circuit activated.

\subsubsection{3-StubTuners}

Connected to an HOM port of a cavity and properly tuned, a 3-stub tuner reflects the HOM voltage back to the cavity with a $180^{\circ}$ phase advance. Because the stub tuner is a passive device, the attenuation in the cables from the HOM ports prevented optimal Q-damping. Additionally, a broken connection in the cable leading to one of the HOM ports created unwanted reflections to the cavity and further decreased the effectiveness of the damping. Nevertheless, a modest decrease (a factor of 1.5) in the $Q$ of the $2106 \mathrm{MHz}$ mode and the corresponding increase in the threshold current were observed. While the effect of increasing the threshold current was only moderate, this setup could easily be optimized by placing the stub tuner closer to the HOM coupler to avoid the effects of attenuation in the cables.

\subsection{Summary of Suppression Techniques}

Several methods for suppressing beam breakup have been successfully demonstrated in the FEL Driver. A summary of the techniques used and their effect on the $2106 \mathrm{MHz}$ mode are given in Table 1. In some sense, we have been treating the symptoms of BBU rather than the problem itself. Clearly strong HOM damping is desired so that BBU can not develop. However, in the event that an HOM is insufficiently damped and causes the instability, then the aforementioned suppression techniques become quite valuable. A brief discussion on the relative merits of each method is in order.

\begin{tabular}{|l|c|c|c|}
\cline { 2 - 4 } \multicolumn{1}{c|}{} & \multicolumn{2}{c|}{ Threshold Current $(\mathrm{mA})$} & \multicolumn{1}{c|}{} \\
\hline \multicolumn{1}{c|}{ Method } & Before Suppression & After Suppression & Effect \\
\hline Damping Circuit & 2.3 & $7.5-10.5$ & $(3.3-4.6) \times \mathrm{I}_{\mathrm{th}}$ \\
\hline 3-Stub Tuner & 2.1 & 3.4 & $1.6 \times \mathrm{I}_{\mathrm{th}}$ \\
\hline Phase Trombone & 2.0 & -7 & Stabilized \\
\hline Local Reflector & 1.8 & 9 & $5 \times \mathrm{I}_{\mathrm{th}}$ \\
\hline Pseudo-Reflector & 1.8 & -17 & Stabilized \\
\hline
\end{tabular}

Table 1: Summary of the effect of BBU suppression techniques applied to the $2106 \mathrm{MHz} \mathrm{HOM}$ in the Jefferson Laboratory FEL. The first two methods involve direct damping of the $Q$ whereas the remaining three are examples of beam optical suppression techniques [CDT05]. 
First, note that the active damping methods work for only a single mode per cavity. This is due to the fact that these methods require extracting a signal from the HOM coupler, of which there is one per cavity. It is also clear that active damping can increase the threshold by orders of a few, but could not completely stabilize the mode. Nonetheless, the attractive feature of Q-damping is that it does not affect the beam optics. On the other hand, the beam optical suppression techniques were able to stabilize the $2106 \mathrm{MHz}$ mode against beam breakup. During the experimental investigations of BBU we were not concerned with establishing a setup with lasing, thus one of the outstanding questions that remain is; does invoking beam optical suppression techniques prohibit one from achieving a machine configuration that is suitable for lasing?

In an effort to provide a more robust BBU suppression technique, the idea of using a beam-based feedback system has been considered [ERL05]. In principle, such a system (which is routinely used in high average current storage rings) could increase the threshold current due to any number of dangerous HOMs and not place any restrictions on the allowable machine optics.

\title{
5.5. Extraction of HOM Power
}

In addition to the beam stability issues, another problem is the excitation of HOMs by high average current, short bunch length beams in the SRF environment. The potentially large HOM power that can be generated extends over very high frequencies (hundreds of $\mathrm{GHz}$ ) and presents an increased cryogenic load due to the power dissipated on the cavity walls [HOMpower]. For example, the monopole mode power excited when a $0.77 \mathrm{~mm}, 77 \mathrm{pC}$ bunch traverses a 9-cell TESLA-type cavity is $185 \mathrm{~W}$. Of this power, approximately $80 \mathrm{~W}$ are at frequencies below $5 \mathrm{GHz}$, while the remaining $105 \mathrm{~W}$ are at frequencies above $5 \mathrm{GHz}$ and up to $100 \mathrm{GHz}$ [LiaEPAC]. The goal is to extract this power in such a way as to maintain good cryogenic efficiency. Consider again the HOM damping scheme that has been adapted for the Cornell ERL. Higher-order mode loop couplers on both sides of an RF cavity are used to couple out modes below approximately $5 \mathrm{GHz}$ and bring them to room temperature loads for absorption whereas ferrite ring-absorbers are used for modes above $5 \mathrm{GHz}$ [Liepe].

\section{SUMMARY}

In this paper, we have considered some of the most outstanding issues related to using SRF linac technology in ERL drivers for light sources. These include ensuring good cryogenic efficiency, operating at high loaded $Q$ in the presence of microphonic and Lorentz force detuning and path length fluctuations. The importance for strong HOM damping to ensure beam stability against beam breakup and efficient extraction of HOM power were also stressed. Many of these issues have been addressed by work performed at the JLab ERL based FEL. In particular, a great deal of progress has been made in the understanding of BBU and practical techniques that can be used to suppress it. Additionally, tremendous progress has been made in the operation of high loaded $Q$ cavities while maintaining adequate phase and amplitude control. While many issues remain, the numerous ERL projects and proposals being pursued around the world will provide a fertile ground for research to resolve and overcome these challenges.

\section{ACKNOWLEDGEMENTS}

The author gratefully acknowledges the following individuals for their helpful discussions while preparing this manuscript: Curt Hovater, Lia Merminga, Tom Powers, and Robert Rimmer. This work was supported by the Office of Naval Research, the Joint Technology Office, the Commonwealth of Virginia, the Air Force Research Laboratory, and by DOE Contract DE-AC05-84ER40150.

\section{REFERENCES}

\author{
[Benson] S. Benson et al., Proc. 2004 FEL Conf., p.229, 2004. \\ [Tigner] \\ [Lia EPAC] \\ [Ann Rev] \\ [White] \\ [BINP] \\ [Un] \\ M. Tigner, Nuovo Cim. 37, p.1228, 1965. \\ L. Merminga, Proc. 2004 Eur. Part. Accel. Conf., p. 16, 2004. \\ L. Merminga, D. Douglas and G. Krafft, Annu. Rev. Nucl. Part. Sci., 53, p.387, 2003. \\ S. Gruner, D. Bilderback, and M. Tigner, http://erl.chess.cornell.edu/papers/WhitePaper_v41.pdf \\ V. Bolotin et al., Proc. 2004 Ru. Part. Accel. Conf., p.33, 2004. \\ G. Krafft and L. Merminga, Unpublished work.
}


[CEBAF] ～C. Leemann, D. Douglas and G. Krafft, Annu. Rev. Nucl. Part. Sci., 51, p.413, 2001.

[ELIC] L. Merminga et al., Proc. 2002 Eur. Part. Accel. Conf., p.203, 2002.

[ERHIC] V. Ptitsyn et al., Proc. 2004 Eur. Part. Conf., p.923, 2004.

[eCOOL] W. MacKay et al., Proc. 2001 Part. Accel. Conf., p.3126, 2001.

[Schneider03] W.J. Schneider, P. Kneisel and C.H. Rhodes, Proc. 2003 Part. Accel. Conf., p.2863, 2003.

[Liepe] M. Liepe, Proc. 2003 SRF Work., 2003.

[Padam] H. Padamsee, J. Knobloch and T. Hays, RF Superconductivity for Accelerators, John Wiley \& Sons, Inc., New York, 1998.

[Jacek03] J. Sekutowicz et al., Proc. 2003 Part. Accel. Conf., p.1395, 2003.

[Curt TN] C. Hovater et al., JLAB Report No. TN-05-031, 2005.

[Schilcher] T. Schilcher, PhD Thesis, Univ. of Hamburg, 1998.

[Davis] K. Davis and T. Powers, JLAB Report No. TN-05-040, 2005.

[Qext Opt] L. Merminga and J. Delayen, JLAB Report No. TN-96-022, 1996.

[Liepe05] M. Liepe, Proc. 2005 Part. Accel. Conf., p.X, 2005.

[Delayen99] J. Delayen, Proc. 1999 Part. Accel. Conf, p.928, 1999.

[piezo1]

[piezo2]

[Liepe03]

[Gluck]

[CT PRST]

[EP PRST]

[Rimmer]

[Phase1]

[Barstow]

[cooling]

[Calaga04]

[Jacek99]

[RandS]

[DD]

[SQEEM]

[CDT05]

[Rotate]

[ERL05]

K. Davis and J. Delayen, Proc. 2003 Part. Accel. Conf., p.1383, 2003.

M. Liepe, W. Moeller and S. Simrock, Proc. 2001 Part. Accel. Conf., p.1074, 2001.

M. Liepe and S. Belomestnykh, Proc. 2003 Part. Accel. Conf, p.1329, 2003.

J. Bisognano, R. Gluckstern, Proc. 1987 Part. Accel. Conf, p.1078, 1987.

C. Tennant, et al., Submitted to Phys. Rev. ST Accel. Beams, 2005.

E. Pozdeyev, Phys. Rev. ST Accel. Beams, 8, 054401, 2005.

R. Rimmer et al., Proc. 2005 Part. Accel. Conf., 2005.

S. Gruner and M. Tigner eds., CHESS Tech. Memo 01-003, JLAB-ACT-01-04, 2001.

B. Barstow, M. Liepe and H. Padamsee, Proc. 2003 SRF Work., 2003.

I. Ben-Zvi et al., Proc. 2003 Part. Accel. Conf., p.39, 2003.

R. Calaga, I. Ben-Zvi and Y. Zhao, Proc. 2004 Eur. Part. Accel. Conf., p. 1120, 2004.

J. Sekutowicz et al., Phys. Rev. ST Accel. Beams, 2, 062001, 1999.

R. Rand and T. Smith, Part. Accel., 11, p.1, 1980.

D. Douglas, JLAB Report No. TN-04-017, 2004.

D. Douglas, JLAB Report No. TN-04-016, 2004.

C. Tennant, et al., Proc. 2005 Part. Accel. Conf, 2005.

D. Douglas, JLAB Report No. TN-04-023, 2004.

[HOMpower]

E. Pozdeyev et. al., Proc. 32 ${ }^{\text {nd }}$ ICFA Work. on ERLs, 2005.

L. Merminga et al., Proc. 2000 Lin. Acc. Conf., p.860, 2000. 